\title{
Reducing Unplanned Extubations in the NICU Following Implementation of a Standardized Approach
}

\author{
Kevin L Crezeé RRT-NPS, Robert J DiGeronimo MD, Marilyn J Rigby DNP NNP-BC, \\ Rick C Carter RRT-NPS, and Shrena Patel MD
}

\begin{abstract}
BACKGROUND: Unplanned extubations (UEs) have been associated with increased ventilator days, risk of infection, cardiopulmonary resuscitation, and resuscitation medication usage. The UE rate in our level 4 NICU is lower than the national average. Efforts to further reduce UE events at our institution led an interdisciplinary group to define steps to eliminate UEs. Steps included: (1) requiring at least 2 care providers at the bedside for movement of an intubated subject; (2) standardizing head and endotracheal tube (ETT) position; (3) defining a set methodology for ETT securing; (4) introducing a postoperative handoff to improve communication; and (5) implementing a post-UE assessment tool. METHODS: A quality improvement initiative reviewed subjects and compared UE rates before (January 2013 to December 2013) and after (January 2014 to December 2014) implementation of UE prevention guidelines. A de-identified data set was used for analysis. RESULTS: $67 \mathrm{UE}$ events were identified with $46 \mathrm{UE}$ events at baseline in 2013 compared with 21 in 2014 post-implementation. This amounted to a $64 \%$ decrease in total UE events (from 46 to 21) $(P<.001)$. Additionally, monthly UE rates decreased $50 \%$ (from 3.8 to 1.9 ), and UE events per 100 ventilator days decreased $53 \%$ (from 1.15 to 0.54 ). CONCLUSIONS: The development of standard guidelines to prevent $\mathrm{UE}$ and a quality review process to track UE provided important information for education and practice change. In our NICU, these changes have significantly improved the UE rate through improved teamwork, accountability, and communication. Key words: neonatology; quality improvement; patient safety; unplanned extubation; neonatal intensive care. [Respir Care 2017;62(8):1030-1035. (c) 2017 Daedalus Enterprises]
\end{abstract}

\section{Introduction}

Unplanned extubation (UE) is the fourth leading cause of an adverse event in the NICU. ${ }^{1}$ A UE is defined as any

\footnotetext{
Mr Crezeé and Mr Carter are affiliated with Respiratory Care Services, Primary Children's Hospital, Salt Lake City, Utah. Dr DiGeronimo is affiliated with Division of Neonatology, Seattle Children's Hospital, Seattle, Washington. Dr Patel is affiliated with the Division of Neonatology, University of Utah, Salt Lake City, Utah. Dr Rigby is affiliated with Neonatal Critical Care Services, Primary Children's Hospital, Salt Lake City, Utah.

Mr Crezeé has disclosed a relationship with Mallinckrodt Pharmaceuticals.

Mr Crezeé presented a version of this paper as an Editors' Choice abstract at the AARC Congress 2015, held November 7-10, 2015, in Tampa, Florida.

Correspondence: Kevin Crezeé RRT-NPS. E-mail: kcrezee@ comcast.net.

Supplementary figure related to this paper is available at http:// www.rcjournal.com.
}

unplanned loss of an endotracheal or tracheostomy tube that is used to provide respiratory support or airway stability. ${ }^{2,3}$

UEs have been associated with increased ventilator days, ${ }^{4,5}$ stay, ${ }^{4,5}$ risk of infection, ${ }^{5-7}$ cardiopulmonary resuscitation, and resuscitation medication usage. A patient who experiences a UE event has the potential for developing hypoxia and hypercarbia. Subsequent treatments to provide ventilation support through resuscitation and reestablishing a patent airway may introduce other complications, such as airway trauma, lung injury, or intraventricular hemorrhage. The UE rate is measured in events/100 ventilator days. ${ }^{8}$ A literature review reported UE rates ${ }^{8}$ in neonates ranging from 0.14 to 5.3, with a mean of 3.5 events/100 ventilator days. The NICU in our institution has historically maintained a UE rate of 1.15-1.76, which

DOI: $10.4187 /$ respcare. $0459810.4187 /$ respcare. 04598 
is lower than the national average. Hospital-wide efforts to reduce serious safety events led an interdisciplinary group to define guidelines in an effort to eliminate UEs in our NICU. The guidelines aimed to change our culture of accepting UEs to a culture focused on preventing all UEs.

\section{Methods}

All data came from the level 4 NICU at Primary Children's Hospital (Intermountain Healthcare) in Salt Lake City, Utah. Institutional review board approval was obtained. Any neonate who experienced a UE between January 2013 and December 2014 was included in the study population. All data were prospectively entered into the respiratory care electronic quality database and then later extracted for analysis. All data used for the study were de-identified. A 2-tailed comparison was used for statistical analysis. During the end of the baseline year (2013), an Airway Security and Care Guideline (Table 1 and Fig. 1) was developed and implemented. A quality improvement huddle assessment tool ${ }^{9}$ (see the supplementary figure at http://www.rcjournal.com) was started to provide additional evaluation of UE events in addition to expert review of all established care processes. Additional collaboration was completed to define and expand the established standards for the postoperative process.

The Airway Security and Care Guideline established the double opposing " $Y$ " cloth tape method as our endotracheal tube (ETT)-securing standard, defining expectations for evaluation and placement for existing patients, all new admits, and postoperative patients. Two care providers were required ${ }^{10}$ for turning, repositioning, or moving patients out of bed when the ventilator tubing or ETT needed to be repositioned. Care providers were defined as any combination of respiratory therapist (RT), registered nurse (RN), medical doctor (MD), or neonatal nurse practitioner (NNP). Head position for chest radiograph was defined as head midline and chin neutral to provide a consistent method of

Table 1. Recommended Guidelines to Reduce Unplanned Extubations

Establish a standardized airway management guidelines detailing ETT and tracheostomy best practices.

Require at least 2 providers when moving patients.

Define standards for head and airway position during chest radiograph. Define ETT tube-securing method and depth.

Define securing of airways for new admits and postoperative patients.

Establish a postoperative handoff to empower RT and anesthesia providers to address airway concerns in a timely manner.

Utilize a UE huddle assessment tool to identify contributing factors.

$\overline{\text { ETT }=\text { endotracheal tube }}$

$\mathrm{RT}=$ respiratory therapist

$\mathrm{UE}=$ unplanned extubation

\section{QUICK LOOK}

\section{Current knowledge}

Current literature does not completely define best practices to prevent an unplanned extubation (UE) from occurring. Studies evaluating ETT securing methods have shown a decrease in UEs. Many elements like head position during chest radiograph, two provider turning have limited evidence. The definition of what constitutes an UE is not defined for patients with a tracheotomy tube.

\section{What this paper contributes to our knowledge}

Implementation of UE prevention guidelines with broadbased parameters significantly reduced the rate of UEs in a level 4 NICU. The development of both a core guideline and quality review process that incorporated the bedside clinicians to boardroom administrators improved the UE rate through team work, accountability, and communication.

evaluating the ETT placement unless contraindicated. The ETT was held in neutral position securely during chest radiograph by a caregiver as defined above wearing protective shielding. Most often, the chest radiograph was completed with the respiratory therapist maintaining the airway. Target ETT depth was defined as mid- to lower trachea. Mid-trachea was defined as half the distance from the carina to the thoracic inlet. ETT security was assessed on admission, and the securing method was changed if loose or different from the defined standard. ETT security was regularly assessed by the RT and RN. Postoperative patients were assessed upon arrival to the NICU by the RT and anesthesia provider to evaluate ETT placement and security. The ETT was moved and/or resecured as needed by the defined guideline. Respiratory therapist and RN staffing was not changed as part of the UE guideline. An average NICU ventilator/RT ratio during this time period was $4-5$ ventilated patients to 1 therapist based on work load. Nurse/patient ratios during this time period ranged on average from 2:1 to 1:2 based upon patient acuity. Difficult airways were identified by the MD or NNP based upon known structural airway anomalies or history of multiple intubation attempts. A brightly colored "Difficult Airway" sticker was placed on the subject's chart, and the information was included in handoff reporting tools.

A UE huddle tool (see the supplementary figure at http:// www.rcjournal.com) was implemented in the intervention year (2014) that required the RT, RN, NNP, and neonatologist to review any UE event that occurred by the end of the 12-h shift. The huddle tool was utilized in post-UE event review by care providers (MD, NNP, RT, and RN) 


\section{Reducing UnPlanNed Extubations IN THE NICU}

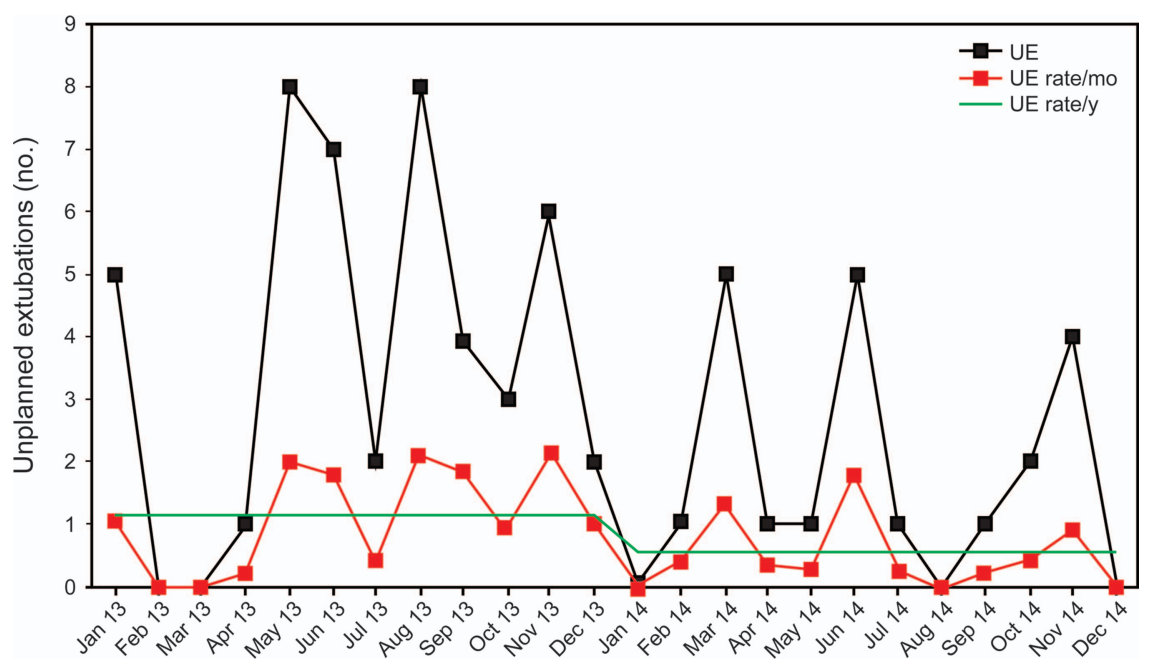

Fig. 1. Trend of unplanned extubation (UE) events from January 2013 to December 2014. The Airway Security and Care Guideline was developed in September 2013, Clinical/medical staff evaluation in January 2014, UE quality improvement huddle assessment tool in February 2014, and collaboration with anesthesia began in March 2014.

Table 2. Group Characteristics

\begin{tabular}{|c|c|c|c|c|c|c|}
\hline & \multicolumn{3}{|c|}{2013} & \multicolumn{3}{|c|}{2014} \\
\hline & Birth GA (Weeks) & UE GA (Weeks) & UE Weight $(\mathrm{kg})$ & Birth GA (Weeks) & UE GA (Weeks) & UE Weight $(\mathrm{kg})$ \\
\hline Minimum & 24 & 29 & 0.81 & 23 & 31 & 0.76 \\
\hline Maximum & 40 & 56 & 5.49 & 38 & 51 & 4.27 \\
\hline Median & 31 & 40 & 2.82 & 29 & 39 & 3.0 \\
\hline \multicolumn{7}{|c|}{$\overline{\mathrm{GA}}=$ gestational age } \\
\hline
\end{tabular}

for adherence to defined standards and risk factor evaluation. The huddle forms were reviewed and analyzed by a UE team comprised of the NICU medical director, associate medical director, NNP manager, and NICU respiratory support nurse and coordinated by the NICU respiratory coordinator. The UE team identified system opportunities for improvement and provided direct and timely feedback to caregivers through event interview/follow-up, newsletters, and presentations. Analyses of data were shared regularly in group communications, quality reports, and presentations at key stakeholder monthly meetings.

\section{Results}

Sixty-seven UE events were identified: 46 UE events in 2013 before implementation and 21 UE events in 2014 after implementation. Contributing factors identified were used in guideline development during the baseline measurement year and were evaluated in the post-implementation year using the UE huddle tool. Table 2 shows group characteristics for birth gestational age, UE gestational age, UE weight in $\mathrm{kg}$ for baseline, and post-intervention years.
Table 3. Activity at Time of Unplanned Extubation

\begin{tabular}{lcr}
\hline \hline \multirow{2}{*}{ Activity } & \multicolumn{2}{c}{$n(\%)$} \\
\cline { 2 - 3 } & 2013 & 2014 \\
\hline Airway/tracheostomy care & $11(24)$ & $10(48)$ \\
Unsecure/loose airway & 0 & $5(24)$ \\
Spontaneous & $11(24)$ & $3(14)$ \\
Procedures/care & $9(19.5)$ & $3(14)$ \\
Other & $15(32.5)$ & 0 \\
\hline
\end{tabular}

Before intervention, $11 \mathrm{UE}$ events (24\%) were associated with airway/tracheostomy care, 11 (24\%) were associated with spontaneous removal of the airway, 9 $(19.5 \%)$ were associated with procedures and care, and $15(32.5 \%)$ were unknown or not clearly identified. After implementation, UE events were most commonly associated with airway/tracheostomy care or pre-airway care for an unsecure or loose airway securement (Table 3).

The quality improvement huddle tool was utilized by the medical care team and providers to evaluate adherence 
Table 4. Huddle Quality Improvement Evaluation Tool Data

\begin{tabular}{lcc}
\hline \hline \multirow{2}{*}{ UE Characteristics } & \multicolumn{2}{c}{$n(\%)$} \\
\cline { 2 - 3 } Time of event: daytime & 2013 & 2014 \\
\hline Male sex & $24(52)$ & $10(48)$ \\
Conventional ventilation & $27(59)$ & $13(62)$ \\
Single UE event & $41(89)$ & $16(76)$ \\
Re-intubation immediately & $31(67)$ & $13(62)$ \\
Re-intubation within 48 h & $29(63)$ & $14(67)$ \\
ETT placed at same depth & $33(72)$ & $16(76)$ \\
Tracheostomy & $24(73)$ & $6(37)$ \\
OG secured to ETT & $6(13)$ & $5(24)$ \\
ETT secure before UE & ND & $5(24)$ \\
Sedation/pain concern & ND & $19(90)$ \\
ETT security concern & ND & $1(5)$ \\
Difficult airway & ND & $9(43)$ \\
Prevention & ND & $2(10)$ \\
& ND & $7(33)$ \\
UE $=$ unplanned extubation & & \\
ETT $=$ endotracheal tube & & \\
OG $=$ orogastric tube & & \\
ND $=$ no data & & \\
\hline
\end{tabular}

Table 5. Post-Unplanned Extubation Support

\begin{tabular}{lcc}
\hline \hline \multirow{1}{*}{ Support } & \multicolumn{2}{c}{$n(\%)$} \\
\cline { 2 - 3 } & 2013 & 2014 \\
\hline Ventilation & $33(72)$ & $16(76)$ \\
NIV & $1(2)$ & $2(9.5)$ \\
Nasal CPAP & $2(4)$ & $1(5)$ \\
HFNC & $1(2)$ & 0 \\
Supplemental oxygen & $9(20)$ & $2(9.5)$ \\
& & \\
NIV $=$ noninvasive ventilation & & \\
HFNC = high-flow nasal cannula & & \\
\hline
\end{tabular}

to the airway care and security guidelines. The huddle group provided direct feedback on the profile of UE for future guideline development, recognition of a UE, and success of resuscitation efforts. Data from the huddle evaluation tool are listed in Table 4. Of interest, a similar number of events occurred during day and night shifts and were more common in infants on conventional versus high-frequency ventilation. Total tracheostomy decannulation UE events decreased slightly year to year. Post-UE, the majority of infants required reintubation and continued invasive ventilator support (Table 5).

Overall, we experienced a $64 \%$ decrease in total UE events from 46 to $21(P<.001)$, resulting in a $50 \%$ decrease in the number of UE events per month (from 3.8 to 1.9 ). The yearly UE rate decreased $53 \%$ from 1.15 to 0.54 per 100 ventilator days (Fig. 1).

\section{Discussion}

We found that implementation of UE prevention guidelines significantly reduced the rate of UEs in a level 4 NICU despite an increase in ventilator days. Our project supports the benefit of encouraging a safety culture to successfully implement measures aimed at improving best practices. In the setting of current literature, which does not completely define best practices to prevent a UE from occurring, this project importantly defines a standard for head positioning ${ }^{11}$ and ETT placement on chest radiograph. The ETT travels greater than $40 \%$ of the length of the trachea with head movement when the chin is moved from the full down to full up position as an example of extension or flexion. ${ }^{9}$ With broad-based parameters, our guideline assists the team members by providing a consistent evaluation tool that helps to reduce practice variation among clinicians. Post-UE in the intervention year, the ETT was more likely to be placed at a location different from that in the pre-UE event (27\% vs 63\%). Most commonly, the ETT post-UE was advanced to the mid- to lower tracheal position.

We also found that defining and standardizing ETT securement was helpful to prevent UEs. It is not clear that any one ETT-securing method is the best. Several studies ${ }^{8}$ have evaluated specific methods, but other care practices utilized in conjunction with various methods are mostly unknown and may play a role in their effectiveness at reducing UEs. We use a conventional double opposing Y method with cloth tape ${ }^{7}$ that was previously evaluated and chosen due to increased UE rates with other methods. Our efforts targeted other methods of airway securing that were previously allowed for periods of time and contributed to our baseline UE events, such as reducing the time between identification of an unsecure airway to resecuring the airway or allowing a non-standard securing method to remain in use for a period of time after admission. We concluded that identifying the need to secure the airway and reeducate ${ }^{10}$ staff to frequently assess airway security was most beneficial. As described by Meyers, ${ }^{2}$ we felt that any unplanned removal of the airway should be considered as a UE. To provide an example, an infant who spontaneously removed his ETT during the ventilator weaning process, even when extubation may have been discussed, but not ordered, was classified as a UE. To exclude these UE events from the total number would mask the actual UE rate and limit the evaluation of clinical practices. Tracheostomy subjects were also included for the same reasons. In response to tracheostomy UE decannulation events, we implemented an evaluation process to consider the appropriateness of a same-diameter-sized longer tracheostomy tube. Establishing a postoperative handoff process partnership with anesthesia providers expanded the opportunity to evaluate ETT placement and security early to ensure that 
airway characteristics and ventilation support were effective. The desired ETT depth in the operating room may differ from the target depth in the NICU due to surgery type and/or positioning. Therefore, we implemented a practice change to ensure that ETTs were adjusted and/or secured upon arrival to the NICU before medical team reporting. This reduced most handoff-related UE events.

ETT security and subject sedation were also important factors that became apparent from the UE huddle. The ETT was inspected as secure $90 \%$ of the time before an unplanned extubation event. Our standards were not set to require inspection at set time intervals. Extended periods of time may have occurred since the ETT security was last assessed. Sedation/pain was reported as a concern in one event in the intervention year using our neonatal pain and sedation score. Neonatal pain and sedation score were reported from the last assessment period but may not reflect the role of sedation in preventing UE events. ${ }^{4}$ After reviewing UE events, the UE group currently recommends the use of procedural sedation when the airway needs to be resecured. ETT security ${ }^{8,9}$ was identified as a concern in $43 \%$ of intervention year UE events. Empowering clinical staff to address airway security concerns early may have contributed to our reduction in UE events from baseline to intervention years. It will remain an ongoing focus along with procedural sedation as we strive to decrease UE events. Capnography was not required in the guideline to be utilized before removing the ETT with a suspected UE event. Capnography was utilized in several events before the ETT was removed but most commonly for ETT placement verification, as is our standard of care if (re-)intubation is needed.

UE events associated with subject movement were reduced from 19.5 to $14 \%$ with introduction of the 2-care provider requirement and increased UE risk education. The majority of UE events occurred during conventional ventilation (from 89 to $76 \%$ ) with fewer events related to high-frequency ventilation. Many factors may influence this relationship, including sedation, different ventilator circuits, the clinical staff perception of a less sick subject, and the percentage of conventional versus high-frequency devices operating at any given time.

We also found that identification of difficult airways was helpful. Difficult airways were identified by the medical staff based upon difficult intubation with multiple attempts or due to known difficult airway anatomy. Ten percent of UEs were identified as difficult airways before the event. Currently, airway signs are placed at the bedside identifying critical airways. As described in the study by Merkel et al, ${ }^{10}$ airway signs may not contribute to a reduction in UEs but instead may provide beneficial information to care providers during post-UE resuscitation.
Finally, we have aimed at improving extubation rates when patients meet criteria. Post-UE, 28 subjects (24\%) were successful at remaining extubated with noninvasive ventilation support or supplemental oxygen beyond $48 \mathrm{~h}$. Current efforts from UE reviews are aimed at reducing the time to extubation to $<1 \mathrm{~h}$ after the extubation guideline is met and a NNP/MD has ordered extubation. One third of UE events in the intervention year were identified by the huddle team as preventable. Huddle feedback has identified that improved adherence to guideline elements could have helped to avoid these UE events. The UE group has expanded the review process to provide feedback to providers who had an opportunity to intervene before the UE event occurred. The feedback identifies specific areas to evaluate when caring for patients and is educationally focused to improve care.

We plan to continue to track our findings and update guidelines to continue our reduction of UEs in the NICU at Primary Children's Hospital. The airway security and care guideline is currently being implemented within Intermountain Healthcare to evaluate the effectiveness in level 3C NICUs across the Intermountain Healthcare system.

\section{Conclusions}

The development of both a core and quality review process provided valuable information for post-event review, education, practice changes, and post-event care. In our NICU, these changes have significantly improved the UE rate through improved teamwork, accountability and communication among members of the clinical medical team.

\section{ACKNOWLEDGMENTS}

We thank the staff and leadership of PCH Respiratory Care Services, NICU, Anesthesia, and Quality for ongoing efforts, support, and guidance to improve patient care.

\section{REFERENCES}

1. Sharek PJ, Horbar JD, Mason W, Bisarya H, Thurm CW, Suresh G, et al. Adverse events in the neonatal intensive care unit: development, testing, and findings of an NICU-focused trigger tool to identify harm in North American NICUs. Pediatrics 2006;118(4):13321340.

2. Meyers JM, Pinheiro J, Nelson MU. Unplanned extubation in NICU patients: are we speaking the same language? J Perinatol 2015;35(9): 676-677.

3. Loughead JL, Brennan RA, DeJuilio P, Camposeo V, Wengert J, Cooke D, et al. Reducing accidental extubation in neonates. Jt Comm J Qual Patient Saf 2008;34(3):164-170, 125.

4. Veldman A, Trautschold T, Weiss K, Fischer D, Bauer K. Characteristics and outcome of unplanned extubation in ventilated preterm and term newborns on a neonatal intensive care unit. Paediatr Anaesth 2006;16(9):968-973.

5. de Lassence A, Alberti C, Azoulay E, Le Miere E, Cheval C, Vincent $\mathrm{F}$, et al. Impact of unplanned extubation and reintubation after wean- 


\section{Reducing UnPlanNed Extubations IN THE NICU}

ing on nosocomial pneumonia risk in the intensive care unit: a prospective multicenter study. Anesthesiology 2002;97(1):148-156.

6. Elward AM, Warren DK, Fraser VJ. Ventilator-associated pneumonia in pediatric intensive care unit patients: risk factors and outcomes. Pediatrics 2002;109(5):758-764.

7. Chuang ML, Lee CY, Chen YF, Huang SF, Lin IF. Revisiting unplanned endotracheal extubation and disease severity in intensive care units. PLoS One 2015;10(10):e0139864.

8. Silva PS, Reis ME, Aguiar VE, Fonseca MC. Unplanned extubation in the neonatal ICU: a systematic review, critical appraisal, and evidence-based recommendations. Respir Care 2013;58(7):12371245.

9. Barber JA. Unplanned extubation in the NICU. J Obstet Gynecol Neonatal Nurs 2013;42(2):233-238.

10. Merkel L, Beers K, Lewis MM, Stauffer J, Mujsce DJ, Kresch MJ. Reducing unplanned extubations in the NICU. Pediatrics 2014;133(5): e1367-e1372.

11. Lange M, Jonat S, Nikischin W. Detection and correction of endotracheal-tube position in premature neonates. Pediatr Pulmonol 2002;34(6):455-461.

This article is approved for Continuing Respiratory Care Education credit. For information and to obtain your CRCE

(free to AARC members) visit 